\title{
GENERAL RELATIVISTIC SIMULATIONS OF MAGNETIZED PLASMAS AROUND MERGING SUPERMASSIVE BLACK HOLES
}

\author{
Bruno Giacomazzo ${ }^{1,2,3}$, John G. BaKer ${ }^{3}$, M. Coleman Miller $^{2,4}$, Christopher S. Reynolds $^{2,4}$, and James R. van Meter ${ }^{3}$ \\ ${ }^{1}$ JILA, University of Colorado and National Institute of Standards and Technology, 440 UCB, Boulder, CO 80309, USA \\ ${ }^{2}$ Department of Astronomy, University of Maryland, College Park, MD 20742, USA \\ ${ }^{3}$ Gravitational Astrophysics Laboratory, NASA Goddard Space Flight Center, Greenbelt, MD 21114, USA \\ ${ }^{4}$ Joint Space Science Institute, University of Maryland, College Park, MD 20742, USA \\ Received 2012 March 28; accepted 2012 April 30; published 2012 May 25
}

\begin{abstract}
Coalescing supermassive black hole binaries are produced by the mergers of galaxies and are the most powerful sources of gravitational waves accessible to space-based gravitational observatories. Some such mergers may occur in the presence of matter and magnetic fields and hence generate an electromagnetic counterpart. In this Letter, we present the first general relativistic simulations of magnetized plasma around merging supermassive black holes using the general relativistic magnetohydrodynamic code Whisky. By considering different magnetic field strengths, going from non-magnetically dominated to magnetically dominated regimes, we explore how magnetic fields affect the dynamics of the plasma and the possible emission of electromagnetic signals. In particular, we observe a total amplification of the magnetic field of $\sim 2$ orders of magnitude, which is driven by the accretion onto the binary and that leads to much stronger electromagnetic signals, more than a factor of $10^{4}$ larger than comparable calculations done in the force-free regime where such amplifications are not possible.
\end{abstract}

Key words: accretion, accretion disks - black hole physics - gravitational waves - magnetohydrodynamics (MHD) - methods: numerical

Online-only material: color figures

\section{INTRODUCTION}

Space-based gravitational-wave (GW) detectors, such as the planned $e$ LISA/NGO and SGO detectors, are expected to detect tens of supermassive black hole $(\mathrm{BH})$ mergers per year. These detections will provide superbly precise measurements of the redshifted masses of the holes as well as the luminosity distance to the event. However, it is not possible to extract the redshift directly from the GWs. For this it is necessary to look for electromagnetic signatures that would identify the host galaxy. The resulting combination of the redshift with the luminosity distance would provide a powerful cosmological probe (Hughes \& Holz 2003; Berti et al. 2005; Kocsis et al. 2006; Arun et al. 2009). It would also allow precise tests of whether GWs travel at the speed of light, as required by general relativity. Although the merger itself produces no electromagnetic emission, if there are significant electromagnetic fields or mass nearby in an accretion disk then there are various possibilities (Schnittman 2011). For some disk accretion rates and binary mass ratios, the binary reaches a point in its coalescence such that further inspiral by emission of GWs occurs more rapidly than the disk diffuses inward (Armitage \& Natarajan 2002; Milosavljević \& Phinney 2005). This leads to a hole in the disk which is filled gradually after merger, leading to a source that brightens over weeks to years depending on various parameters (Armitage \& Natarajan 2002; Milosavljević \& Phinney 2005; Krolik 2010; Tanaka \& Menou 2010; Shapiro 2010). Several authors have discussed consequences of the recoils from asymmetric emission of GWs during the coalescence, from prompt shocks to delayed emission lasting millions of years (Shields \& Bonning 2008; Schnittman \& Krolik 2008; Megevand et al. 2009; Lippai et al. 2008; Corrales et al. 2010; Anderson et al. 2010; Rossi et al. 2010; Zanotti et al. 2010). Emission might occur in the late inspiral from effects such as enhanced accretion, periodic Newtonian perturbations, or shearing of the disk due to GWs (Kocsis \&
Loeb 2008). Earlier precursors are also possible, and in some cases the error volume from the GW signal may be small enough that the host galaxy can be identified by morphology, mass, or by the presence of an active galactic nucleus.

In the last few years there have been a number of publications describing the evolution of gas and magnetic fields around merging supermassive BHs. van Meter and collaborators performed test-particle simulations of the motion of accreting gas during the last phase of inspiral of comparable-mass supermassive BHs (van Meter et al. 2010). These simulations suggested that near merger a significant fraction of particles can collide with each other at speeds approaching the speed of light, implying that a burst of radiation might accompany the coalescence. Other works have instead started to investigate the effect that the merging BHs would have on surrounding gas and the possible emission of electromagnetic signals (O'Neill et al. 2009; Farris et al. 2010, 2011; Bode et al. 2010; Bogdanović et al. 2011; Bode et al. 2012). At the same time there have been the first investigations of the effect of binary black hole (BBH) mergers on electromagnetic fields in vacuum (Palenzuela et al. 2009, 2010c; Mösta et al. 2010) and in a magnetically dominated plasma (Palenzuela et al. 2010a, 2010b; Mösta et al. 2012).

These studies have shown how magnetic and electric fields can be distorted by the motion of the BHs and hence lead to possible electromagnetic emission. In particular, recent studies by Palenzuela and Mösta (Palenzuela et al. 2010a, 2010b; Mösta et al. 2012) have raised the possibility that the motion of two BHs in a magnetically dominated plasma, i.e., in the so-called force-free regime, could generate two separate jets, one around each $\mathrm{BH}$, during the inspiral. At the time of the merger these two collimated jets would enter in contact and form a single jet emitted from the spinning $\mathrm{BH}$ formed after the merger. It is, however, still unknown how general this scenario is and whether the emission would be detectable. 
In this Letter, we present the first results from general relativistic magnetohydrodynamic (GRMHD) simulations of magnetized plasmas around merging supermassive black holes. By considering the evolution of equal-mass BBH systems in plasmas with different levels of magnetization we fill the gap between the studies of non-magnetized gas and the results obtained in the force-free and electro-vacuum regimes. We use a spacelike signature $(-,+,+,+)$ and will typically use a system of units in which $c=G=M=1$, where $M$ is the total mass of the binary. In these units $1 M$ is equivalent to $\sim 0.14 M_{8} \mathrm{hr}$ and to $\sim 4.86 \times 10^{-6} M_{8}$ pc, where $M_{8} \equiv M /\left(10^{8} M_{\odot}\right)$.

\section{NUMERICAL METHODS AND INITIAL DATA}

Most of the details on the mathematical and numerical setup used for producing the results presented here are discussed in depth elsewhere (Pollney et al. 2007; Thornburg 2004; Giacomazzo \& Rezzolla 2007; Giacomazzo et al. 2009, 2011; Löffler et al. 2012). In what follows, we limit ourselves to a brief overview.

\subsection{Magnetohydrodynamics and Einstein Equations}

The evolution of the spacetime was obtained using the Ccatie code, a three-dimensional finite-differencing code providing the solution of a conformal traceless formulation of the Einstein equations (Pollney et al. 2007), and we used the "moving puncture" method and gauge conditions developed in van Meter et al. (2006). The GRMHD equations were instead solved using the Whisky code (Giacomazzo \& Rezzolla 2007; Giacomazzo et al. 2011), which adopts a flux-conservative formulation of the equations as presented in Antón et al. (2006) and high-resolution shock-capturing schemes. All the results presented here have been computed using the piecewise parabolic method, while the Harten-Lax-van Leer-Einfeldt approximate Riemann solver has been used to compute the fluxes (Giacomazzo \& Rezzolla 2007). All the simulations were performed using a polytropic equation of state (EOS) with a polytropic exponent $\gamma=4 / 3$ and a polytropic constant $\kappa=0.2$. We used a polytropic EOS instead of an ideal-fluid EOS because the computation of primitive from conservative variables in highly magnetized plasmas is much simpler and more robust for polytropes (see Giacomazzo \& Rezzolla 2007 for details). The main results of our work are unaffected by this choice.

In order to guarantee the divergence-free character of the MHD equations, we evolve the vector potential as described in Giacomazzo et al. 2011. When evolving the vector potential a gauge choice needs to be made and we here use the "algebraic gauge" (Etienne et al. 2012), which was also used in previous GRMHD simulations with the Whisky code (Giacomazzo et al. 2011; Rezzolla et al. 2011). The code has been validated against a series of tests in special relativity (Giacomazzo \& Rezzolla 2006) and in full general relativity (Giacomazzo \& Rezzolla 2007).

Since the simulations performed here consider a plasma with a total mass negligible with respect to the mass of the two BHs, we have decoupled the Einstein equations from the matter dynamics, i.e., the metric variables are evolved using Einstein equations in vacuum. The same was done in the general relativistic hydrodynamic simulations reported in Farris et al. (2010). Moreover, in order to prevent the formation of nonphysical values in the MHD quantities, we have excised the MHD variables inside the apparent horizon of each $\mathrm{BH}$.

\subsection{Adaptive Mesh Refinement}

Both the Einstein and the GRMHD equations are solved using the vertex-centered adaptive-mesh-refinement (AMR) approach provided by the Carpet driver (Schnetter et al. 2004). Our rather basic form of AMR consists of centering the highest-resolution level around each $\mathrm{BH}$ and in moving the "boxes" following the position of the two BHs. For the results presented here we have used 11 refinement levels with the finest resolution being $0.0375 M$ and the coarsest resolution being $38.4 M$. The finest grid has a radius of $6 M$ whereas the coarsest grid extends to $1536 \mathrm{M}$. The large extent of our finest grid allows us to follow accurately the dynamics of the plasma around the BHs and it is also sufficiently large to avoid the spurious magnetic field amplifications that may occur when evolving the vector potential with the "algebraic gauge" (Etienne et al. 2012).

For all the simulations reported here we have also used a reflection-symmetry condition across the $z=0$ plane and a $\pi$-symmetry condition across the $x=0$ plane. $^{5}$

\subsection{Initial Data}

We have considered equal-mass systems of two non-spinning BHs with an initial separation of $8.48 \mathrm{M}$; these inspiral for approximately three orbits before merger. The initial data were computed using the public available TwoPuncture code developed in Ansorg et al. (2004) and we chose the momentum of the punctures in order to ensure that the orbit of the two $\mathrm{BHs}$ is quasicircular. We have considered two models: B0 is surrounded by a non-magnetized plasma, and model B2 instead has an initially uniform magnetic field with a ratio of magnetic to gas pressure $\left(p_{\text {mag }} / p_{\text {gas }} \equiv \beta^{-1}\right)$ initially equal to $2.5 \times 10^{-2}$. The magnetic field is aligned with the total angular momentum of the system, i.e., $B^{i}=\left(0,0, B^{z}\right)$ while in all the models the rest-mass density $\rho$ is initially uniform and fills the entire domain; we have in mind a flow that could be advection-dominated close to the holes (e.g., Ichimaru 1977) and thus have a high enough radial velocity that it can keep up with the binary inspiral throughout the entire coalescence. The initial distribution of the magnetic field is similar to that adopted in previous works (Palenzuela et al. 2010a, 2010b; Mösta et al. 2012) and it assumes that the magnetic field is anchored to a circumbinary disk located far outside of the numerical domain. We note that these initial data are similar to what was used in previous non-magnetic (Farris et al. 2010) and force-free (Palenzuela et al. 2010a, 2010b; Mösta et al. 2012) analyses. After this Letter was submitted, Noble et al. 2012 presented preliminary results describing the evolution of a magnetized circumbinary accretion disk up to few orbits before the $\mathrm{BH}$ merger. Their simulations showed that magnetized plasma can indeed flow in the region where the BHs merge, and in future simulations we plan to use initial conditions more similar to the end result of the simulations recently reported in Noble et al. (2012).

\section{DYNAMICS}

Figure 1 shows the evolution of the rest-mass density $\rho$ on the equatorial plane for models B0 (left panels) and B2 (right panels). The evolution of the non-magnetized model (left panels) resembles the one described in Farris et al. (2010) with the formation of two spiral shocks during the inspiral and the formation of a central spinning $\mathrm{BH}$ surrounded by a spherical

\footnotetext{
5 Stated differently, we evolve only the region $[x \geqslant 0, z \geqslant 0]$ and apply a $180^{\circ}$-rotational-symmetry boundary condition across the plane at $x=0$.
} 

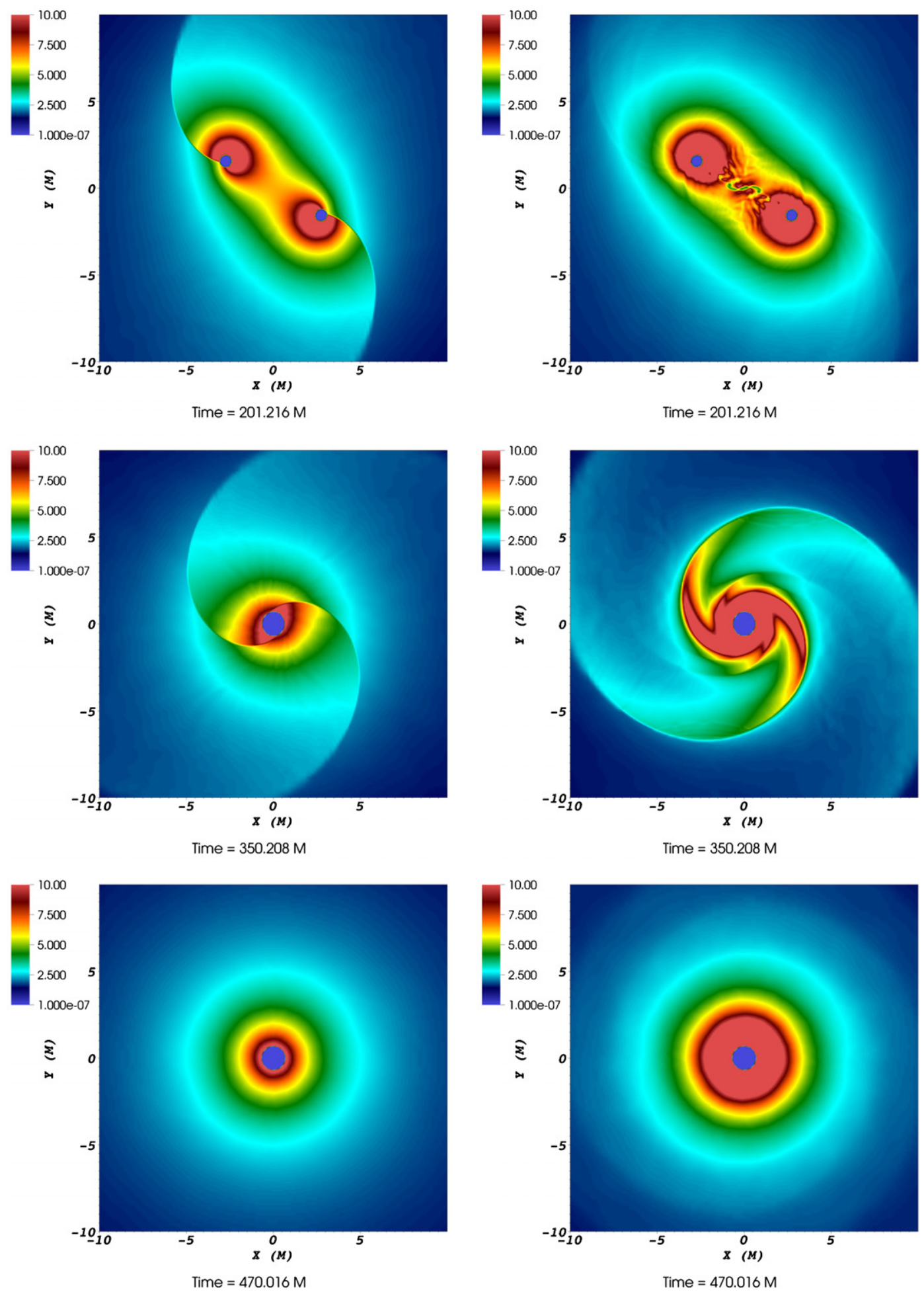

Figure 1. Evolution of the rest-mass density $\rho$ on the equatorial plane for the non-magnetized model B0 (left panels) and for the magnetized case B2 (right panels) The units of time (shown at the bottom of each panel) and distance are $M$ and the rest-mass density is normalized to its initial value.

(A color version of this figure is available in the online journal.)

distribution of matter accreting onto it. The magnetized model (right panels) shows quite different dynamics. During the evolution the magnetic field strength increases by approximately two orders of magnitude and contributes significantly to the total pressure in the gas. Because of this, ${ }^{6}$ the two shock waves that

\footnotetext{
6 See Mizuno et al. (2009) for an example of how the magnetic pressure can
} affect shock formation. are present during the inspiral of model B0 are strongly reduced and hardly visible (first panel in the right column). Moreover, the density close to each of the two BHs and in the region connecting the two BHs is much larger than in the unmagnetized case. In this region it is also possible to see the formation of instabilities that are not present without magnetic fields. Soon after the merger (second panel in the right column) the spinning $\mathrm{BH}$ is surrounded by a disk with a density a factor of $\sim 3$ larger 

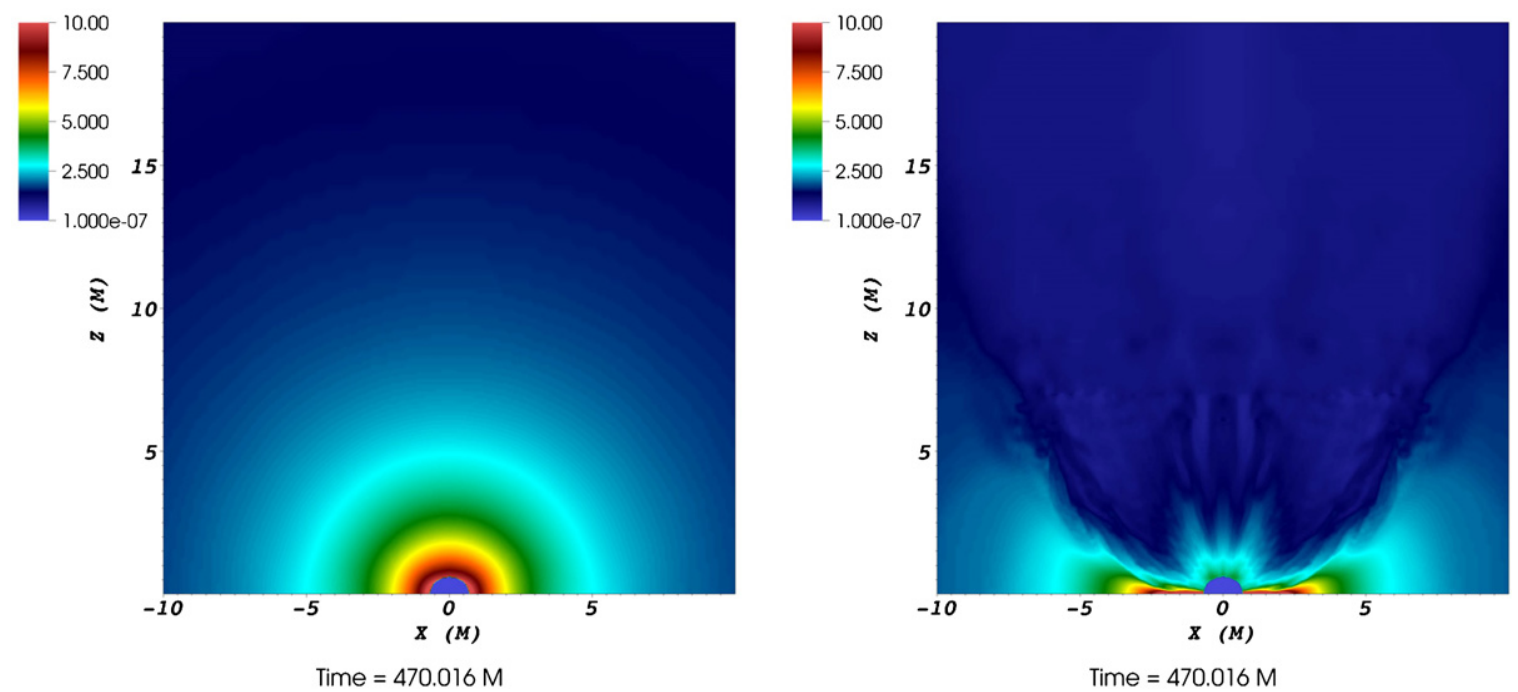

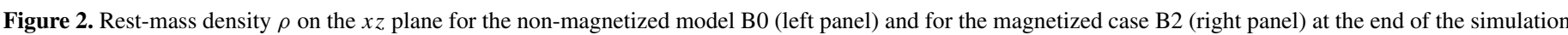
$(t \sim 470 M)$. The unit of distance is $M$ and the rest-mass density is normalized to its initial value.

(A color version of this figure is available in the online journal.)
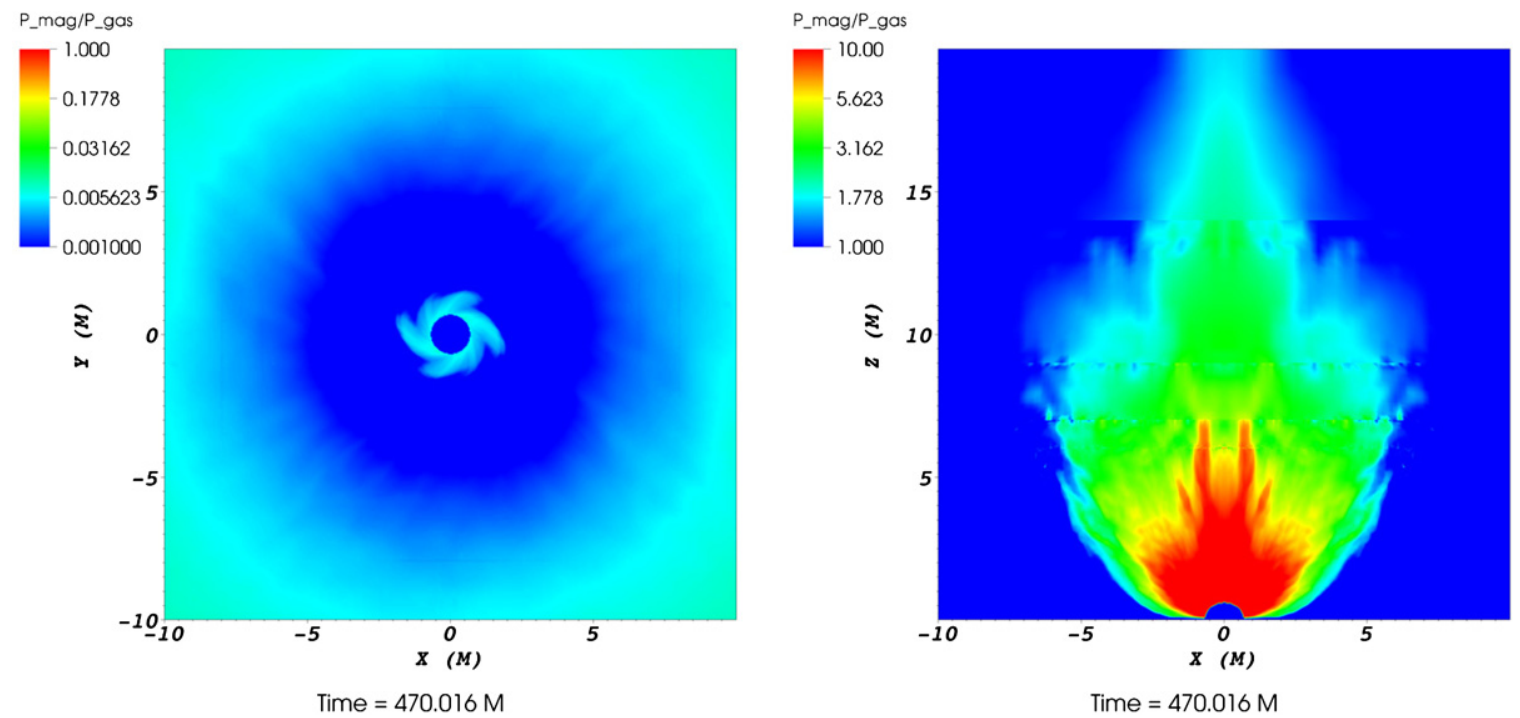

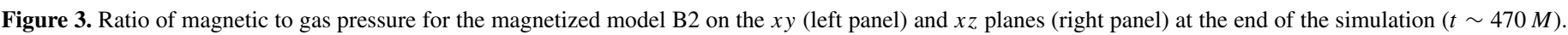

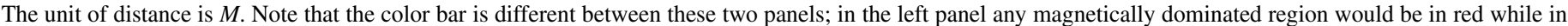

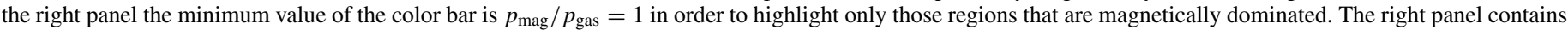
five refinement levels; the finest refinement extends to $z=6$ and the coarsest applies to $z=14-20$.

(A color version of this figure is available in the online journal.)

than in the unmagnetized case, and two shock waves are formed and the system finally relaxes to its final configuration (third panel in the right column). The temperature in the magnetic simulation is larger by up to $\sim 40 \%$ for model B2 than in the unmagnetized model.

Although the evolution on the equatorial plane already shows some differences due to the effect of the magnetic field, the main difference is in the rest-mass density on the meridional plane. In Figure 2 we show the rest-mass density $\rho$ on the $x z$ plane for models B0 (left panel) and B2 (right panel) at the end of the simulation $(t \sim 470 M)$. Whereas the end result of the evolution of the unmagnetized model is a plasma accreting spherically onto a spinning $\mathrm{BH}$, in the case of the magnetized model B2 the system forms a thin accretion disk and a funnel is created around the spin axis of the $\mathrm{BH}$. Although at this time no relativistic jet is emitted (the Lorentz factor is lower than $\sim 3$ at the end of the simulation), such emission might exist at later times (which are outside the scope of the present Letter).

The difference between the rest-mass density distribution in the unmagnetized and magnetized cases can be better understood by looking at Figure 3, which shows the ratio of magnetic to gas pressure (i.e., the inverse of the plasma parameter $\beta$ ) for the magnetized model B2 on the $x y$ (left panel) and $x z$ planes (right panel) at the end of the simulation $(t \sim 470 M)$. On the equatorial plane no region is magnetically dominated and the central region inside the disk has larger values of $\beta$ than in the initial conditions, but the $x z$ plane shows clearly the presence of a strongly magnetically dominated region close to the spin axis of the $\mathrm{BH}$ with $\beta \sim 10^{-2}$. During the inspiral and merger magnetic field lines are indeed compressed and twisted causing the magnetic field to be amplified of approximately two orders of magnitude. This highly magnetized region is responsible for 

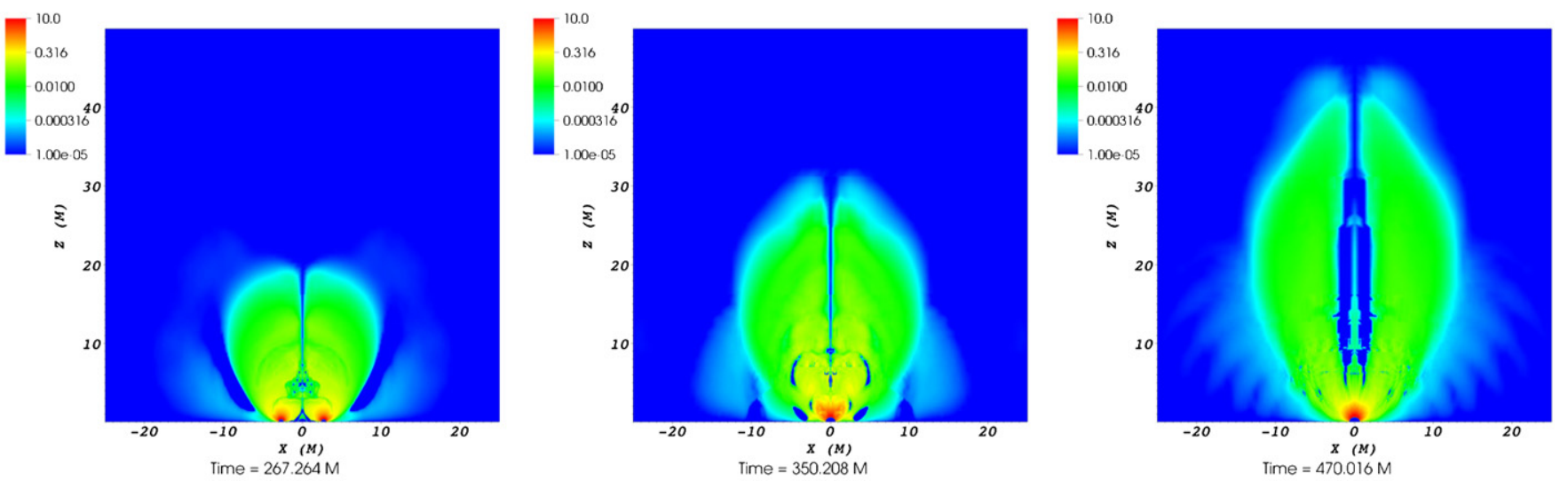

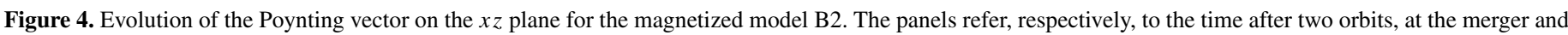
at the end of the simulation. The units of time (shown at the bottom of each panel) and distance are $M$.

(A color version of this figure is available in the online journal.)

the creation of the thin disk and its funnel. If these simulations had been run for many orbital times (which would have required much larger computational resources), the amplification of the magnetic field would likely have been even larger, due to effects like the magnetorotational instability (Balbus \& Hawley 1991).

\section{ELECTROMAGNETIC EMISSION}

In Figure 4 we show the evolution of the $z$ component of the Poynting vector on the $x z$ plane for model B2. We show in particular its outgoing component after two orbits (first panel), at the time of the merger (second panel) and at the end of the simulation (third panel). One of the main differences with respect to force-free simulations is that we do not observe the two strong and distinct jets originating from each $\mathrm{BH}$ and the "jet" that is emitted by the system propagates slowly into the medium surrounding the binary. Whereas in the force-free scenarios the jet propagates in a very low-density medium where the inertia of the plasma can be neglected, in our simulations the jet has to break through the infalling medium outside the binary. We also note that in our scenario the emission is mainly collimated and parallel to the angular momentum of the binary and to the spin of the final $\mathrm{BH}$ with no sign of the dominant quadrupolar emission that was observed in the recent force-free simulations of Mösta et al. (2012). We also note that the noncollimated emission discovered in Mösta et al. (2012) is larger than their collimated emission and it is $\sim 600$ times smaller than our luminosity.

Finally, in Figure 5 we show the Poynting flux luminosity computed for model B2 (blue solid line). We have rescaled our results to consider a binary system with a total mass of $10^{8} M_{\odot}$ and immersed in a plasma with a rest-mass density $\rho=10^{-11} \mathrm{~g} \mathrm{~cm}^{-3}$. This corresponds to having an initial magnetic field of $\sim 10^{4} \mathrm{G}$ for model B2, which is also the same magnetic field strength considered in Palenzuela et al. (2010b). The luminosity is computed at a distance of $z=10 \mathrm{M}$. Model B2 shows the characteristic increase in luminosity during the inspiral, with a peak corresponding to the time of the merger, followed by a drop-off of a factor $\sim 2$. This is qualitatively similar to what is observed in force-free simulations (Palenzuela et al. 2010b), but our actual luminosities are considerably higher. This happens because in our ideal GRMHD simulations the magnetic field is amplified of $\sim 2$ orders of magnitude. So even when starting with a magnetic field of $\sim 10^{4} \mathrm{G}$, the final configuration has a field of $\sim 10^{6} \mathrm{G}$. If we were to compare

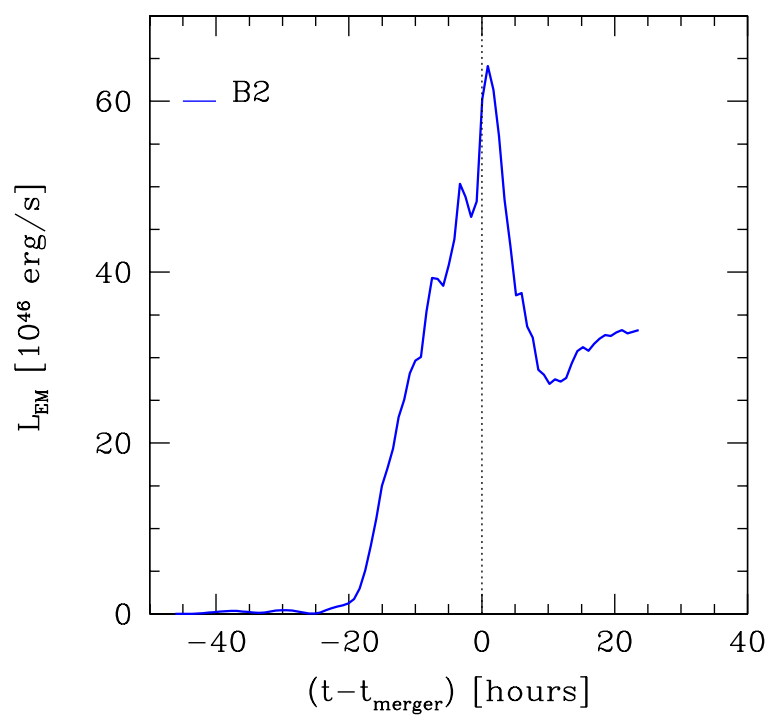

Figure 5. Evolution of the luminosity for the magnetized model B2 (blue solid line). The luminosity is computed at a distance $z=10 M$ for a binary system with a total mass of $10^{8} M_{\odot}$, an initial rest-mass density of $10^{-11} \mathrm{~g} \mathrm{~cm}^{-3}$, and an initial magnetic field of $\sim 10^{4} \mathrm{G}$.

(A color version of this figure is available in the online journal.)

with force-free simulations starting with such a high field we would obtain similar luminosities (Palenzuela et al. 2010b). Indeed, we note that our luminosity at the end is $\sim 10^{47} \mathrm{erg} \mathrm{s}^{-1}$ and in the simulations reported in Palenzuela et al. (2010b) is $\sim 10^{43} \mathrm{erg} \mathrm{s}^{-1}$ for a magnetic field that is $\sim 2$ orders of magnitude lower than the one we have at the end of our simulations. Since the luminosity $L_{\mathrm{EM}}$ scales as $B^{2}$ our values are consistent with those one would observe in a force-free regime. This again highlights one of the main differences between our simulations and those assuming a force-free regime since the beginning. Because of the accretion of the plasma onto the BHs, magnetic field lines are compressed and twisted driving the large amplification we observe. In a force-free regime, the magnetic field is decoupled from the dynamics of the matter and such large amplifications cannot be obtained.

\section{CONCLUSIONS}

We have presented the first numerical GRMHD simulations of magnetized plasmas around merging supermassive BBHs. We 
have for the first time investigated the role of magnetic fields in the plasma dynamics and filled the gap between the works that have considered non-magnetized gas and the results obtained in the force-free and electro-vacuum regimes.

We have shown that even plasmas that are initially not magnetically dominated have different dynamics than in the unmagnetized case and that magnetic plasmas can generate strong and collimated electromagnetic emission. We therefore generalize the physical regimes of matter and electromagnetic fields around coalescing $\mathrm{BHs}$ that can lead to potentially detectable emission.

We thank Phil Armitage, Tamara Bogdanovic, Bernard Kelly, Krzysztof Nalewajko, Carlos Palenzuela, Luciano Rezzolla, Jeremy Schnittman, and Roman Shcherbakov for useful comments and suggestions. We also thank Philip Cowperthwaite for help in visualizing some of the numerical data. Resources supporting this work were provided by the NASA High-End Computing (HEC) program through the NASA Advanced Supercomputing (NAS) Division at Ames Research Center and NASA Center for Climate Simulation (NCCS) at Goddard Space Flight Center. Numerical simulations were also performed on the cluster RANGER at the Texas Advanced Computing Center (TACC) at The University of Texas at Austin through XSEDE grant No. TG-PHY110027. B.G. acknowledges support from NASA grant No. NNX09AI75G and NSF grant No. AST 1009396. J.B. and J.v.M. acknowledge support from NASA grant 09-ATP090136.

\section{REFERENCES}

Anderson, M., Lehner, L., Megevand, M., \& Neilsen, D. 2010, Phys. Rev. D, 81,044004

Ansorg, M., Brügmann, B., \& Tichy, W. 2004, Phys. Rev. D, 70, 064011

Antón, L., Zanotti, O., Miralles, J. A., et al. 2006, ApJ, 637, 296

Armitage, P. J., \& Natarajan, P. 2002, ApJ, 567, L9

Arun, K. G., Mishra, C. K., Van Den Broeck, C., et al. 2009, Class. Quantum Grav., 26, 094021

Balbus, S. A., \& Hawley, J. F. 1991, ApJ, 376, 214

Berti, E., Buonanno, A., \& Will, C. M. 2005, Class. Quantum Grav., 22, 943

Bode, T., Bogdanovic, T., Haas, R., et al. 2012, ApJ, 744, 45

Bode, T., Haas, R., Bogdanović, T., Laguna, P., \& Shoemaker, D. 2010, ApJ, 715,1117
Bogdanović, T., Bode, T., Haas, R., Laguna, P., \& Shoemaker, D. 2011, Class. Quantum Grav., 28, 094020

Corrales, L. R., Haiman, Z., \& MacFadyen, A. 2010, MNRAS, 404, 947

Etienne, Z. B., Paschalidis, V., Liu, Y. T., \& Shapiro, S. L. 2012, Phys. Rev. D, 85,024013

Farris, B. D., Liu, Y. T., \& Shapiro, S. L. 2010, Phys. Rev. D, 81, 084008

Farris, B. D., Liu, Y. T., \& Shapiro, S. L. 2011, Phys. Rev. D, 84, 024024

Giacomazzo, B., \& Rezzolla, L. 2006, J. Fluid Mech., 562, 223

Giacomazzo, B., \& Rezzolla, L. 2007, Class. Quantum Grav., 24, S235

Giacomazzo, B., Rezzolla, L., \& Baiotti, L. 2009, MNRAS, 399, L164

Giacomazzo, B., Rezzolla, L., \& Baiotti, L. 2011, Phys. Rev. D, 83, 044014

Hughes, S. A., \& Holz, D. E. 2003, Class. Quantum Grav., 20, 65

Ichimaru, S. 1977, ApJ, 214, 840

Kocsis, B., Frei, Z., Haiman, Z., \& Menou, K. 2006, ApJ, 637, 27

Kocsis, B., \& Loeb, A. 2008, Phys. Rev. Lett., 101, 041101

Krolik, J. H. 2010, ApJ, 709, 774

Lippai, Z., Frei, Z., \& Haiman, Z. 2008, ApJ, 676, L5

Löffler, F., Faber, J., Bentivegna, E., et al. 2012, Class. Quantum Grav., 29, 115001

Megevand, M., Anderson, M., Frank, J., et al. 2009, Phys. Rev. D, 80, 024012

Milosavljević, M., \& Phinney, E. S. 2005, ApJ, 622, L93

Mizuno, Y., Zhang, B., Giacomazzo, B., et al. 2009, ApJ, 690, L47

Mösta, P., Alic, D., Rezzolla, L., Zanotti, O., \& Palenzuela, C. 2012, ApJ, 749, L32

Mösta, P., Palenzuela, C., Rezzolla, L., et al. 2010, Phys. Rev. D, 81, 064017

Noble, S. C., Mundim, B. C., Nakano, H., et al. 2012, arXiv:1204.1073

O’Neill, S. M., Miller, M. C., Bogdanović, T., Reynolds, C. S., \& Schnittman, J. D. 2009, ApJ, 700, 859

Palenzuela, C., Anderson, M., Lehner, L., Liebling, S. L., \& Neilsen, D. 2009, Phys. Rev. Lett., 103, 081101

Palenzuela, C., Garrett, T., Lehner, L., \& Liebling, S. L. 2010a, Phys. Rev. D, 82,044045

Palenzuela, C., Lehner, L., \& Liebling, S. L. 2010b, Science, 329, 927

Palenzuela, C., Lehner, L., \& Yoshida, S. 2010c, Phys. Rev. D, 81, 084007

Pollney, D., Reisswig, C., Rezzolla, L., et al. 2007, Phys. Rev. D, 76, 124002

Rezzolla, L., Giacomazzo, B., Baiotti, L., et al. 2011, ApJ, 732, L6

Rossi, E. M., Lodato, G., Armitage, P. J., Pringle, J. E., \& King, A. R. 2010, MNRAS, 401, 2021

Schnetter, E., Hawley, S. H., \& Hawke, I. 2004, Class. Quantum Grav., 21, 1465

Schnittman, J. D. 2011, Class. Quantum Grav., 28, 094021

Schnittman, J. D., \& Krolik, J. H. 2008, ApJ, 684, 835

Shapiro, S. L. 2010, Phys. Rev. D, 81, 024019

Shields, G. A., \& Bonning, E. W. 2008, ApJ, 682, 758

Tanaka, T., \& Menou, K. 2010, ApJ, 714, 404

Thornburg, J. 2004, Class. Quantum Grav., 21, 743

van Meter, J. R., Baker, J. G., Koppitz, M., \& Choi, D.-I. 2006, Phys. Rev. D, 73,124011

van Meter, J. R., Wise, J. H., Miller, M. C., et al. 2010, ApJ, 711, L89

Zanotti, O., Rezzolla, L., Del Zanna, L., \& Palenzuela, C. 2010, A\&A, 523, A8 